\title{
Evaluation of BMI and Lipids Profile in Type 2 Diabetic Subjects with Low and Raised Levels of Thyroid Hormone in Calabar, Nigeria
}

\author{
C. E. J. Udiong1 ${ }^{*}$, M. H. Etukudoh ${ }^{2}$, I. K. Isong2, E. F. Udoisa ${ }^{3}$ \\ ${ }^{1}$ Department of Chemical Pathology, Faculty of Medicine and Dentistry, College of Medical Sciences, University \\ of Calabar, Calabar, Nigeria \\ ${ }^{2}$ Department of Medical Laboratory Science, Faculty of Allied Medical Science, University of Calabar, Calabar, \\ Nigeria \\ ${ }^{3}$ Luciama Specialist Hospital, Ikot Efanga, Calabar, Nigeria \\ Email: "chriudiong@yahoo.com
}

Received 28 July 2015; accepted 2 November 2015; published 5 November 2015

Copyright @ 2015 by authors and Scientific Research Publishing Inc.

This work is licensed under the Creative Commons Attribution International License (CC BY).

http://creativecommons.org/licenses/by/4.0/

(c) (i) Open Access

\section{Abstract}

Relationship between lipid levels and BMI was investigated in euthyroid, hyperthyroid, hypothyroid, general Type 2 diabetics, and non-diabetic control subjects. FT $_{4}, \mathrm{~T}_{4}, \mathrm{~T}_{3}$ and TSH did not differ in obese and non-obese diabetics but were higher in diabetics than in non-diabetics $(p=0.015$, $0.012,0.0164$ respectively). Levels of $\mathrm{FT}_{4}$ correlated with TC, LDL-C, VLDL-C, TG and HDL-C ( $\mathrm{r}=$ $0.179, p=0.034 ; r=0.183, p=0.033 ; r=0.183 p=0,033 ; r=0.176, p=0.037 ; r=-0.210, p=0.023$ respectively). $T_{3}$ levels correlated with TC $(r=0.210: p=0.023)$, LDL-C $(r=0.193: p=0.025)$, and VLDL-C ( $\mathrm{r}=0.244: p=0.003)$ levels in diabetic subjects. $\mathrm{FT}_{4}$ and $\mathrm{T}_{4}$ levels correlated with BMI only in the non-diabetic subjects $(p=0.022,0.025)$ respectively. TC and LDL-C levels correlated positively with BMI in non-diabetics $(r=0.265, p=0.006 ; r=0.249, p=0.010)$, general diabetics $(r=$ $0.247, p=0.016 ; r=0.291, p=0.002)$, euthyroid $(r=0.274, p=0.017 ; r=0.252, p=0.022)$ and hyperthyroid $(\mathrm{r}=0295, p=0.008 ; \mathrm{r}=0.346, p=0.002)$ diabetics respectively. There were negative correlations between BMI and HDL-C in the diabetics $(r=-0.188, p=0.018)$ and euthyroid $(r=$ $-0.273, p=0.018$ ) groups. Atherogenic index correlated negatively with BMI in all 5 groups of subjects. The coexistence of both low and raised levels of thyroid hormones in the diabetic population altered the levels and interrelationship between lipids and BMI. Altered levels of FT 4 observed in Type 2 diabetics emanated from diabetes not obesity. Atherogenic index is an efficient indicator of atherogenic risk irrespective of complications.

\footnotetext{
"Corresponding author.
}

How to cite this paper: Udiong, C.E.J., Etukudoh, M.H., Isong, I.K. and Udoisa, E.F. (2015) Evaluation of BMI and Lipids Profile in Type 2 Diabetic Subjects with Low and Raised Levels of Thyroid Hormone in Calabar, Nigeria. Journal of Diabetes Mellitus, 5, 277-283. http://dx.doi.org/10.4236/jdm.2015.54034 
Keywords

Diabetes, Hypothyroid, Hyperthyroid, Lipids, BMI

\section{Introduction}

The presence of abnormal thyroid hormone levels in diabetic subjects without symptoms of thyroid gland dysfunction has been reported by many researchers [1]-[5]. The need to carry out routine thyroid function test on diabetics has also been recommended particularly on diabetic subjects whose conditions are difficult to manage [3] [6]. The influence of raised or low thyroid hormone level on Body mass index (BMI), lipid levels and on the relationship between BMI and thyroid hormones, BMI and lipids, and between thyroid hormones and lipids has not been defined in diabetic subjects. The exact effect, magnitude and direction of such influence are poorly known. Information on the relationship among lipids namely, Total Cholesterol (TC), High Density Lipoprotien Cholesterol (HDL-C), Low Density Lipoprotien Cholesterol (LDC-C), Very Low Density Lipoprotien Cholesterol (VCDL-C) Triglyceride (TG) and BMI in euthyroid, hypothyroid and hyperthyroid Type 2 diabetics is not defined. This study sought to evaluate the influence if any of the co-existence of abnormal thyroid hormone levels on BMI and lipids in diabetic subjects.

\section{Materials and Methods}

The study was carried out on diabetic subjects who were attending routine diabetic clinics. The diabetic subjects selected showed no clinical signs of thyroid disorders. Informed Consent was obtained from the study subjects and co-operation was received from the patients' physicians. The samples were sourced from University of Calabar Teaching Hospital, University of Calabar Medical Centre and some private clinics in Calabar, Cross River State of Nigeria. All diabetic subjects were confirmed diabetics who previously had or were having plasma glucose levels of $6.1 \mathrm{mmol} / 1$ or higher more than once and were receiving treatment for hyperglycaemia. The heights of the patients were measured using a height meter in meter $(\mathrm{m})$ while their weights were measured using clinical weighing balance in $\mathrm{kg}$. BMI was calculated from the height and weight of each subject thus.

$$
\mathrm{BMI}=2 \frac{\text { Weight in kg }}{\text { (Height in metre })}[7]
$$

The initial criteria used for the selection of Type 2 diabetics from Type 1 were age of onset of diabetes mellitus (age greater than 35 years) and non dependence on insulin alone to achieve normal plasma glucose levels. Diabetics who met the above criteria and fasting C-peptide level of above $0.38 \mathrm{mg} / \mathrm{ml}$ were classified as type 2 [5]. Seven milliliters $(7 \mathrm{ml})$ of fasting blood samples were collected from each diabetic and control subjects who were age and sex marched non-diabetic subjects selected from the same community as the diabetic subjects. The collected blood was dispensed into a plain sample bottle (without anticoagulant) and was allowed to stand and clot before serum was harvested from it. The harvested serum was divided into three aliquots and stored frozen. Each aliquot was thawed once only when needed. ELISA kit for C-peptide, total thyroxine $\left(\mathrm{T}_{4}\right)$, free thyroxine $\left(\mathrm{FT}_{4}\right)$, total triiodothyronine $\left(\mathrm{T}_{3}\right)$ and thyroid stimulating hormone (TSH) reagents kits were obtained from DSLab Inc. California, USA and were used for the assay of C-peptide and the thyroid hormones. Fax stat plate reader and washer were used to process the assays. Lipid profile kits (TC, LDL-C, VLDL-C, HDL-C and triglyceride) were products of Biolabo Fimes, SA, France. Glucose was estimated using the method of Trinder [8]. Biotec spectrophotometer was employed for absorbance measurements.

Quality control sera (Seratex) were used to monitor the performance of each parameter. Initial recovery experiments were performed on each of thyroid function tests (TSH, $\mathrm{FT}_{4}, \mathrm{~T}_{4}$ and $\mathrm{T}_{3}$ ) to assess the accuracy of the methods. Within and between batch variations analysis was performed. The results were within the ranges recommended by manufacturers of the reagents kits.

Data analysis was performed using t-test on a two-tailed distribution using SPSS programme while correlation analysis and plots were performed using Pearson correlation coefficient formula using Microsoft excel programme. 


\section{Result}

The subjects were divided into 5 groups based on diabetes status and thyroid hormone levels as follows; nondiabetic controls $(0.78-2.20 \mathrm{ng} / \mathrm{dl})$, all Type 2 diabetic subjects $(<0.78->2.2 \mathrm{ng} / \mathrm{dl})$, those with euthyroid levels $(0.78-2.20 \mathrm{ng} / \mathrm{dl})$, those with low levels $(<0.78 \mathrm{ng} / \mathrm{dl}$, hypothyroid) and those with raised levels $(>2.20 \mathrm{ng} / \mathrm{dl}$, hyperthyroid). Among the 162 type 2 diabetic subjects studied 33 (20.30\%) were obese and 129 (79.7\%) were non-obese. Comparison of the level of thyroid hormones in the obese and non-obese showed no significant difference between the two groups (TSH $p=0.260 ; \mathrm{FT}_{4} p=0.580, \mathrm{~T}_{4} p=0.130, \mathrm{~T}_{3} p=0.178$ ), as shown in Table 1. The levels of TC, TG, LDL-C were significantly higher in diabetics than in non-diabetic control subjects $(p=$ $<0.0001,0.020,<0.0001$ respectively), while HDL-C was significantly lower in diabetics than in the control subjects. In Table 2, TC correlated positively with $\mathrm{FT}_{4}$ and $\mathrm{T}_{4}$ in the all Type 2 diabetics $(\mathrm{r}=0.179, p=0.034: \mathrm{r}$ $=0.210, p=0.023$ respectively). Positive correlations were observed between $\mathrm{FT}_{4}$ and LDL-C $(\mathrm{r}=0.183, p=$ $0.033)$, VLDL-C $(\mathrm{r}=0.183, p=0.033)$ and TG $(\mathrm{r}=0.176, p=0.037)$. HDL-C correlated negatively with $\mathrm{FT}_{4}(\mathrm{r}$ $=-0.210, p=0.023)$. There were also positive correlation between $\mathrm{T}_{3}$ and TC $(\mathrm{r}=0.210, p=0.023)$; LDL-C (r $=0.193, p=0.025)$, and VLDL-C $(\mathrm{r}=0.244, p=0.003)$.

Correlation analysis between BMI and TSH showed no significant association in non-diabetic controls, General diabetics, hypothyroid and hyperthyroid diabetics. Correlation analysis between BMI and both $\mathrm{FT}_{4}$ and $\mathrm{T}_{4}$ showed significant association only in the non-diabetic subjects $(\mathrm{r}=0.225, p=0.022 ; \mathrm{r}=0.220, p=0.025)$. There were no significant correlations between BMI and $\mathrm{FT}_{4}, \mathrm{~T}_{4}$ or $\mathrm{T}_{3}$ in non-diabetic controls, all Type 2 diabetics, euthyroid, hypothyroid and hyperthyroid diabetics as presented in Table 3. BMI correlated positively with TC and LDL-C respectively in non-diabetics controls $(\mathrm{r}=0.265, p=0.006: \mathrm{r}=0.349, p=0.010)$; all Type 2 diabetics $(\mathrm{r}=0.247, p=0.016: \mathrm{r}=0.0291 p=0.002)$ euthyroid $(\mathrm{r}=0.274, p=0.017: \mathrm{r}=0.252, p=0.022)$,

Table 1. Comparison of thyroid hormone levels in A: diabetic and non-diabetic control and B: obese and non-diabetic subjects.

\begin{tabular}{ccccccc}
\hline & $\begin{array}{c}\text { A } \\
\text { Diabetic }\end{array}$ & Non-diabetic controls & & $\begin{array}{c}\text { B } \\
\text { Obese diabetics }\end{array}$ & Non-obese diabetics \\
\hline N & 162 & 105 & & 33 & 129 & \\
\hline T-test analysis & $\mathrm{M}+\mathrm{SD}$ & $\mathrm{M}+\mathrm{SD}$ & $p$ & $\mathrm{M}+\mathrm{SD}$ & $\mathrm{M}+\mathrm{SD}$ & $p$ \\
\hline TSH MIU/MI & $1.80 \pm 1.62$ & $2.34 \pm 1.24$ & $0.0164^{*}$ & $2.80 \pm 4.49$ & $1.84 \pm 1.62$ & 0.260 \\
FT4 ng/1 & $1.62 \pm .72$ & $1.19 \pm 0.46$ & $0.015^{*}$ & $2.08 \pm 2.16$ & $1.30 \pm 1.78$ & 0.580 \\
T4 $\mu \mathrm{g} / \mathrm{dl}$ & $9.09 \pm 5.19$ & $7.68 \pm 2.06$ & $0.012^{*}$ & $7.95 \pm 4.05$ & $8.42 \pm 3.37$ & 0.130 \\
T3 ng/dl & $112 \pm 88$ & $110 \pm 46$ & 0.277 & $136 \pm 119$ & $110 \pm 46$ & 0.178 \\
\hline
\end{tabular}

$\mathrm{TSH}=$ Thyroid Stimulating Hormone; $\mathrm{FT}_{4}=$ Free thyroxine; $\mathrm{T}_{4}=$ Total Thyroxine; $\mathrm{T}_{3}=$ Total Triiodothyronine; $\mathrm{N}=$ Number; ${ }^{*}$ significant.

Table 2. Correlation analysis between thyroid hormones and lipids in type 2 diabetic subjects.

\begin{tabular}{ccc}
\hline Subjects & All type 2 diabetics & $p$ \\
N & 162 & $0.034^{*}$ \\
Analysis & $\mathrm{r}$ & $0.023^{*}$ \\
TC vs. FT4 & 0.179 & $0.023^{*}$ \\
TC vs. T3 & 0.21 & $0.033^{*}$ \\
HDL-C vs. FT4 & -0.21 & $0.025^{*}$ \\
LDL-C vs. FT4 & 0.183 & $0.033^{*}$ \\
LDL-C vs. T3 & 0.193 & $0.003^{*}$ \\
VLDL-C vs. FT4 & 0.244 & 0.037 \\
VLDL-C vs. T3 & 0.224 & 0.071 \\
TG vs. FT4 & 0.176 & 0.151 \\
TG vs. T3
\end{tabular}

$\mathrm{TC}=$ Total cholesterol; FT4 = Free Thyroxine; T3 = Total triiodothyronine; HDL-C = high density ipoprotein cholesterol; LDL-C = low density lipoprotein cholesterol; VLDL-C $=$ Very low density lipoprotein cholesterol; $\mathrm{TG}=$ Triglyceride; $\mathrm{N}=$ Number of subjects; ${ }^{*}=$ significant. $^{2}$ 
Table 3. Correlation analysis between BMI and thyroid hormones in non-diabetic, general diabetics, euthyroid, hypothyroid and hyperthyroid diabetic subjects.

\begin{tabular}{ccccccccccc}
\hline Subjects & Non-diabetic & Control & Type 2 & Diabetics & Euthyroid & Diabetics & Hypothyroid & Diabetics & Hyperthyroid Diabetics \\
\hline Analysis & $\mathrm{r}$ & $p$ & $\mathrm{r}$ & $p$ & $\mathrm{r}$ & $p$ & $\mathrm{r}$ & $p$ & $\mathrm{r}$ & $p$ \\
BMI vs. TSH & 0.06 & 0.079 & 0,064 & 0.418 & 194 & 0.055 & 0.069 & 0.704 & 0.061 & 0.591 \\
BMI vs. FT $_{4}$ & 0.225 & 0.022 & 0.032 & 0.51 & 0.0013 & 00.901 & 0.024 & 0.334 & 0.158 & 0.16 \\
BMI vs. T & 0.22 & 0.025 & 0.11 & 0.134 & 0.104 & 0.303 & 0.025 & 0.804 & 0.073 & 0.712 \\
BMI vs. T & 0.025 & 0.803 & 0.14 & 0.169 & 0.111 & 0.27 & 0.025 & 0.83 & 0.168 & 0.133 \\
Cut off & $0.78-$ & $2.2 \mathrm{ng} / \mathrm{dl}$ & ------- & ------- & $0.78-$ & $2.20 \mathrm{ng} / \mathrm{dl}$ & $<0.78 \mathrm{ng} / \mathrm{dl}$ & & $>2.20 \mathrm{ng} / \mathrm{dl}$ & \\
\hline
\end{tabular}

$\mathrm{BMI}=$ Body Mass Index; $\mathrm{T}_{4}=$ Total Thyroxine; $\mathrm{TSH}=$ Thyroid Stimulation Hormone; $\mathrm{FT}_{4}=$ Free Thyroxine; $\mathrm{T}_{3}=$ Total Triiodothyronine; $\mathrm{NS}=\mathrm{No}$ Significant; ${ }^{*} \mathrm{~S}=$ Significant; ${ }^{* *}=$ Strongly significant.

hyperthyroid $(\mathrm{r}=0.295, p=0.008 ; \mathrm{r}=0.346, p=0.002)$. BMI also correlated negatively with HDL-C (Table 4) in the all Type 2 diabetics $(\mathrm{r}=0.188, p=0.018)$ and euthyroid $(\mathrm{r}=-0.273, p=0.017)$ subjects. Similar correlations in non-diabetic and hypothyroid diabetics were not significant. There was no significant correlation between BMI and VLDL-C on one hand and TG on the other hand. Significant negative correlations were observed between BMI and the atherogenic index HDL-C/LDL-C in the control, all diabetics, euthyroid, hyperthyroid and hypothyroid diabetic subjects $(\mathrm{r}=-0.510, p=0.0001 ; \mathrm{r}=-0.475, p=0.00012 ; \mathrm{r}=-0.233, p=0.019 ; \mathrm{r}=$ $-0.304, p=0.006, \mathrm{r}=-0238, p=0.013$ respectively as presented in Table 4 .

\section{Discussion}

Thyroid disorders, dyslipidemia and obesity are severally reported in diabetes mellitus [6] [9] [10]. Obesity is said to predispose individuals to diabetes [9] while dyslipidemia is associated with both obesity and diabetes mellitus [11]. Recently there has been a call on researchers to determine whether hypothyroidism causes obesity or obesity causes hypothyroid [12]. In this study we did not find any significant differences between the levels of thyroid hormones in obese and non-obese diabetic subjects (Table 1), even though the levels of those thyroid hormones; $\mathrm{FT}_{4}$, and $\mathrm{T}_{4}$ were significantly higher in diabetic subjects than in non-diabetic controls (Table 5).

This observation portrays diabetes mellitus as the primary cause of altered thyroid hormone levels in diabetic subjects, since the increase in thyroid hormones is found in diabetics irrespective of body weight. This is in agreement with Banduska-Stankiewicz [13] who stated that the changes of thyroid hormone concentration may be regarded as a consequence rather than the cause of obesity.

Expectedly, the levels of TC, LDL-C and TG were significantly higher in diabetic than in non-diabetic control subjects implying that the observed dyslipidemia derived from diabetes mellitus. Unlike the relationship between BMI and the thyroid hormones, each of FT4, $\mathrm{T}_{4}$, and $\mathrm{T}_{3}$ positively correlated with TC, LDL-C, VLDL-C and TG in the type 2 diabetic subjects. Here the impact of thyroid hormone $\mathrm{FT}_{4}, \mathrm{~T}_{4}$ and $\mathrm{T}_{3}$ levels is obviously to increased synthesis or degradation of lipids in order to bring about the observed correlation [14]. This is supported by an existing notion that thyroid hormones control energy homeostasis, influences body weight, thermogenesis, lipolysis and metabolism of cholesterol [13]. Since the levels of thyroid hormone in obese and nonobese did not differ significantly, and BMI did not correlate with thyroid hormones in diabetic subjects it may be reasoned that the correlations observed between BMI and the lipids (TC, LDL-C VLDL-C and TG) were related to thyroid hormones not BMI. The output of such lipids would be predicted by body weight (BMI) as observed in the correlations between TC, LDL-C, HDL-C, TG and BMI (Table 4). The implication is that BMI is only remotely associated with thyroid hormone levels via cholesterol levels. It has been reported that non insulin dependent diabetics often have quantitative change in lipid profile characterized by high triglyceridemia and low HDL-C in average population [10]. This report is in agreement with our observation of low HDL-C and raised triglyceride levels. We also observed significant negative correlation between FT4 and HDL-C suggesting that the rate of HDL-C metabolism is related to free thyroxine.

We did not observe any significant association between BMI and TSH in the non-diabetic controls, all diabetics, hypothyroid and hyperthyroid diabetics. There was a weak positive association only in the euthyroid diabetics, which is in agreement with the opinion of Pearce [12] that there is increased output of TSH in diabetic subjects. Increase plasma TSH has been associated with other non thyroidal hormones such as adiponectin and 
Table 4. Correlation analysis between BMI and lipids in euthyroid, hypothyroid and hyperthyroid diabetics, general diabetic and non-diabetic control subjects.

\begin{tabular}{|c|c|c|c|c|c|c|c|c|c|c|}
\hline \multirow{3}{*}{$\begin{array}{c}\text { Subjects } \\
\text { NO } \\
\text { Analysis }\end{array}$} & \multicolumn{2}{|c|}{ Non-diabetic controls } & \multicolumn{2}{|c|}{ All type 2 diabetics } & \multicolumn{2}{|c|}{ Euthyroid diabetics } & \multicolumn{2}{|c|}{$\begin{array}{l}\text { Hyperthyroid } \\
\text { diabetics }\end{array}$} & \multicolumn{2}{|c|}{$\begin{array}{l}\text { Hypothyroid } \\
\text { diabetics }\end{array}$} \\
\hline & \multicolumn{2}{|r|}{105} & \multicolumn{2}{|c|}{162} & \multicolumn{2}{|c|}{87} & \multicolumn{2}{|c|}{43} & \multicolumn{2}{|c|}{32} \\
\hline & $\mathrm{r}$ & $p$ & $\mathrm{r}$ & $p$ & $\mathrm{r}$ & $p$ & $\mathrm{r}$ & $p$ & $\mathrm{r}$ & $p$ \\
\hline BMI vs. TC & 0.265 & $0.006^{* *}$ & 0.247 & $0.0168^{*}$ & 0.274 & $0.017^{*}$ & 0.295 & $0.008^{* *}$ & 0.169 & 0.278 \\
\hline BMI vs. HDL-C & 0.034 & 0.734 & -0.188 & $0.018^{*}$ & -0.273 & $0.017^{*}$ & 0.065 & 0.652 & 0.034 & 0.780 \\
\hline BMI vs. LDL-C & 0.249 & $0.010^{*}$ & 0.291 & $0.002^{* *}$ & 0.252 & $0.0228^{*}$ & 0.346 & $0.002^{* *}$ & 0.002 & 0.992 \\
\hline BMI vs. VLDL-C & 0.023 & 0.686 & 0.025 & 0.790 & 0.173 & 0.095 & 0.032 & 0.686 & 0.025 & 0.792 \\
\hline BMI vs. HDL-L/LDL-L & -0.510 & $<-0.0001^{* *}$ & -0.475 & $<0.001^{* *}$ & -0.304 & $0.006^{* *}$ & -0.304 & $0.006^{* *}$ & -0.238 & $0.013^{*}$ \\
\hline BMI vs. TG & 0.072 & 0.812 & 0.034 & 0.720 & 0.068 & 0.501 & 0.069 & 0.384 & 0.005 & 0.960 \\
\hline Cut off point & \multicolumn{2}{|c|}{$\mathrm{FT}_{4}=0.78-2.20 \mathrm{ng} / \mathrm{dl}$} & \multicolumn{2}{|r|}{. } & \multicolumn{2}{|c|}{$\mathrm{FT}_{4}=0.78-2.20 \mathrm{ng} / \mathrm{dl}$} & \multicolumn{2}{|c|}{$\mathrm{FT}_{4}<0.78 \mathrm{ng} / \mathrm{dl}$} & \multicolumn{2}{|c|}{$\mathrm{FT}_{4}>2.20 \mathrm{ng} / \mathrm{dl}$} \\
\hline
\end{tabular}

$\mathrm{BMI}=$ Body Mass Index $\left(\mathrm{Wt} / \mathrm{Ht}^{2}\right) ; \mathrm{Wt}=$ Weight in $\mathrm{Kg} ; \mathrm{Ht}^{2}=$ Height in meter square; $\mathrm{M}=$ mean; $\mathrm{SD}=$ standard deviation; $\mathrm{TC}=\mathrm{Total} \mathrm{Cholesterol}$; HDL-C = High Density lipoprotein cholesterol; LDL-C = Low density Lipoprotein cholesterol; LDL-C = Very low density Lipoprotein cholesterol; ${ }^{*}=$ Significant; ${ }^{* *}=$ Highly significant.

Table 5. Plasma lipids in diabetic and non-diabetic subjects.

\begin{tabular}{cccc}
\hline Test & Diabetic & Non-diabetic & $p$ \\
\hline Analysis & $\mathrm{M} \pm \mathrm{SD}$ & $\mathrm{M} \pm \mathrm{SD}$ & $<0.001^{* *}$ \\
\hline $\mathrm{TC} \mathrm{mmol} / 1$ & $6.26 \pm 162$ & $105 \pm 0.83$ & $0.020^{*}$ \\
$\mathrm{TG} \mathrm{mmol} / 1$ & $1.14 \pm 0.57$ & $1.05 \pm 0.49$ & $0.018^{*}$ \\
$\mathrm{HDLC} \mathrm{mmol} / 1$ & $1.20 \pm 0.37$ & $-1.38 \pm 0.47$ & $<0.001^{* *}$ \\
$\mathrm{C} \mathrm{mmol} / 1$ & $4.4 \pm 0.80$ & $3.62 \pm 0.80$ & \\
\hline
\end{tabular}

$\mathrm{M}=$ Mean; $\mathrm{SD}=$ Standard deviation; $\mathrm{TC}=$ Total Cholesterol; $\mathrm{TG}=$ Triglyceride; HDL-C $=$ High density lipoprotein cholesterol; $\mathrm{LDL}-\mathrm{C}=\mathrm{Low}$ Cholesterol; VLDL-C = Very low density lipoprotein cholesterol; $\mathrm{mmol} / \mathrm{l}=$ Millimole per litre; $\mathrm{t}=\mathrm{T}$-test; ${ }^{*}=$ Significant; ${ }^{* *}=$ Highly significant.

leptin [11]. The presence of both low and raised levels of thyroid hormones in the general diabetes population could have masked the increase in TSH level expected in the all diabetic population. There were positive associations between both $\mathrm{FT}_{4}$ and $\mathrm{T}_{4}$ and BMI only in the non-diabetic control subjects. The direction of association in the all Type 2, euthyroid, hyperthyroid and hypothyroid diabetic subjects differed (Table 3). This finding suggests that Type 2 diabetes may trigger a change in plasma thyroxine levels in hypothyroid and hyperthyroid diabetics, probably in response to energy requirement. This reasoning agrees with the report that attributes increased peripheral hormone levels usually found in the upper normal range in obese subjects to adaptation process toward increase energy need in order to reduce body weight [11]. $\mathrm{T}_{4}$ and $\mathrm{T}_{3}$ showed no significant association with BMI in any of the 5 groups of subject (Table 3), an observation that is probably influenced by falsely altered levels of measured total thyroxine emanating from increased carrier proteins commonly found in Type 2 diabetes in association with obesity. For this reason it had been recommended that only $\mathrm{FT}_{4}$ assay should be used for the assessment of thyroid status in diabetics [15]. Total $\mathrm{T}_{4}$ and $\mathrm{T}_{3}$ have been shown to be influenced by extra-thyroid factors [16]. $\mathrm{T}_{3}$ levels are altered in liver diseases and other metabolic disorders [12]. Raised levels of $\mathrm{FT}_{4}$ and $\mathrm{T}_{3}$ are usually associated with decreased total cholesterol levels in non-diabetic subjects [17]. The situation in diabetic subjects may be different in the light of diabetic complications. Thyroid hormone therapy in obese hypothyroid rats failed to decrease fat when treated with $\mathrm{T}_{3}$ [12] indicating that thyroid hormone does not actually influence obesity associated with hypothyroidism.

The relationship between BMI and lipids varied with thyroid hormone levels. Correlation analysis between BMI and TC, LDL-C, VLDL-C and triglycerides in non-diabetic controls, all type 2 diabetic subjects, euthyroid, hyperthyroid and hypothyroid diabetics showed divergent results and patterns (Table 4). BMI correlated positively with TC and LDL-C in non-diabetics, general Type 2, euthyroid and hyperthyroid diabetic subjects. There were no significant associations in the hypothyroid diabetics, meaning that in the absence of adequate thyroid 
hormone cholesterol failed to increase or decrease with BMI but did so in the presence of normal or raised thyroid hormone. It may thus be inferred that, it is thyroid hormones levels rather than BMI that do vary with cholesterol levels, because BMI did not correlate with $\mathrm{FT}_{4}$ in diabetic subjects (Table 3). HDL-C which was inversely correlated with BMI in all Type 2 and euthyroid diabetics, showed no significant association in the control subjects, hypothyroid and hyperthyroid diabetics indicating that the change in HDL-C level is not related to thyroid hormone. In non insulin dependent diabetes (NIDDM) low plasma HDL-C levels are present and this does not seem to be related to control or mode of treatment [18]. Other factors inherent in Type 2 diabetes may be responsible, since the relationship between thyroid disorders and diabetes mellitus is characterized by a complex interdependent interaction [10] [15]. VLDL-C and TG levels did not correlate with BMI in any of the 5 groups of subjects probably because of the varying effect of insulin on both lipoprotein lipase and hepatic triglyceride lipase that innervate their metabolism [19]. The effect of Type 2 diabetes on metabolism of VLDL-C is diverse [20]. Efficient VLDL-C catabolism is associated with low plasma triglyceride concentrations and maximal availability of surface components for transfer to HDL-C [18] [20], conditions that are not present in Type 2 diabetes mellitus. Rather the combination of insulin resistance, hyperinsulinemia and increased free fatty acid flux in NIDDM set the stage for increased secretion of VLDL-C [21] [22]. Our observation is another complication of Type 2 diabetes mellitus. The direction of altered association between BMI and total cholesterol and LDL-C levels is related to thyroid hormone levels. Positive correlations observed between BMI and both TC and LDL-C in the euthyroid and hyperthyroid together with the a negative insignificant association in hypothyroid diabetic subjects culminated in a positive significant association in the all Type 2 diabetic population obviously determined by the number of subjects and the extent of divergence in each group (Table 4). Type 2 diabetes mellitus is characterized by dyslipidemia the level of which is associated with BMI. Raised lipid levels would then trigger corresponding rise in thyroid hormone level. This observation seems to be a compensatory process that is at variance with what obtains in non-diabetic hyperthyroidism [15].

The atherogenic index HDL-C/LDL-C correlated negatively with BMI in the non-diabetics, general, euthyroid, hypothyroid and hyperthyroid diabetics thus emphasizing the risk associated with obesity irrespective thyroid hormone levels, diabetes, non-diabetes or lipid levels.

\section{Summary}

Obesity is not the primary cause of thyroid hormone disorders in type 2 diabetic subjects, because thyroid hormone levels do not differ in obese and non-obese subjects. Rather, thyroid hormones levels correlate with cholesterol levels showing that changes in thyroid hormone levels are related to cholesterol levels. Cholesterol levels in turn correlate with BMI indicating that lipid levels are influenced by BMI. The implication is that BMI is only remotely associated with thyroid hormone levels via cholesterol levels. $\mathrm{FT}_{4}$ did not correlate with BMI in any of the 4 groups of diabetic subjects but correlated in the non-diabetic controls. The coexistence of both low and raised levels of thyroid hormones in the diabetic population altered the levels and interrelationship between lipids and BMI. The extent and direction of variance would depend on the number of subjects and magnitude of change in each group of euthyroid, hypothyroid and hyperthyroid diabetics present in the study population. It is recommended that thyroid hormone levels and BMI be taken into consideration when interpreting cholesterol results in Type 2 diabetic subjects.

\section{References}

[1] Smithson, M.J. (1998) Screening for Thyroid Dysfunction in a Community of Diabetic Patients. Diabetics Medicinal, 15, 148-150. http://dx.doi.org/10.1002/(SICI)1096-9136(199802)15:2<148::AID-DIA540>3.0.CO;2-H

[2] Radetti, G., Paganini, C., Gentili, L., Babin, F., Pasquino, B. and Zachinann, M. (1994) Altered Adrenal and Thyroid Function in Children with Insulin Dependent Diabetes Mellitus. Acta Diabetologia, 31, 128-140. http://dx.doi.org/10.1007/BF00570367

[3] Tunbridge, W.M.G., Evered, D.C. and Hall, R. (1977) The Spectrum of Thyroid Diseases in a Community, the Wickhen Survey. Clinical Endocrinology, 7, 481-493. http://dx.doi.org/10.1111/j.1365-2265.1977.tb01340.x

[4] Udiong, C.E.J., Udoh, A.E. and Etukudoh, H.E. (2007) Evaluation of Thyroid Function in Diabetes Mellitus in Calabar, Nigeria. Indian Journal of Clinical Biochemistry, 22, 74-78. http://dx.doi.org/10.1007/BF02913318

[5] Celani, M.F., Bonati, M.E. and Stucci, N. (1994) Prevalence of Abnormal Thyrotropin Concentration Measured by a Sensitive Assay in Patients with Type 2 Diabetes Mellitus. Diabetes Research, 27, 15-25. 
[6] Udiong, C.E.J., Etukudoh, H.E. and Essien, O.E. (2007) Lipid Profile and Thyroid Hormones in Type 1 and Type 2 Diabetics. Journal of Medical Laboratory Science, 16, 59-67.

[7] WHO (1999) Definition, Diagnosis and Classification of Diabetes Mellitus and Its Complication. Report of WHO Part1: Diagnosis and Classification of Diabetes Mellitus. World Health Organization, Department of Non Communicable Disease Surveillance, Geneva.

[8] Trinder, P. (1969) Determination of Glucose in Blood Using Glucose Oxidase with an Alternative Oxygen Acceptor. Annals of Clinical Biochemistry, 6, 24-27. http://dx.doi.org/10.1177/000456326900600108

[9] Devi, M.A., Singh, N.S. and Singh, S.S. (2013) Thyroid Dysfunction in Type 2 Diabetic Patients in Urban Area of Minipur. International Journal of Pharmaceutical Invention, 2, 7-9.

[10] Peros, P., McCrimmon, R.J., Shaw, G. and Frier, B.M. (1995) Frequency of Thyroid Dysfunction in Diabetic Patients; Value of Annual Screening. Diabetic Medicine, 12, 622-627. http://dx.doi.org/10.1111/j.1464-5491.1995.tb00553.x

[11] Dias, C.M., Noqueirap, P., Rosa, A.V., de Sa, J.V., Gouvea, M.F. and Falcao, J.C.M. (1995) Total Cholesterol and High Density Lipoprotein Cholesterol in Patients with Non-Insulin Dependent Diabetes Mellitus. Acta Médica Portuguesa, 8, 619-628.

[12] Nelson, D. (2012) Hypothyroidism and Obesity. Gastric Surgeon.com. http://www.gastricsurgeon.com/articleshypothyroidism-and-obesity

[13] Pearce, E.N. (2012) Thyroid Hormone and Obesity. Current Opinion in Endocrinology Diabetes and Obesity, 19, 408413. http://dx.doi.org/10.1097/MED.0b013e328355cd6c

[14] Whitley, R.J. (1999) Thyroid Function. In: Burtis, C.A. and Ashwood, E.R., Eds., Text Book of Clinical Chemistry, 3rd Edition, W.B. Saunder Company, Philadelphia, 1496.

[15] Bandursk-Stankiewicz, E. (2013) Thyroid Hormones Obesity and Metabolic Syndrome. Proceedings of the 4th Congress of Polish Thyroid Association, Lodz, 11-13 April 2013, A5. http://dx.doi.org/10.1186/1756-6614-6-s2-a5

[16] Suzukki, J., Nannom, H., Gemma, R., Tanaka, I., Taminato, T. and Yoshimi, T. (1994) The Mechanism of Thyroid Hormone Abnormalities in Patients with Diabetes Mellitus. Folia Endocrinologica Japonica, 70, 465-470. http://dx.doi.org/10.1507/endocrine1927.70.4_465

[17] Strissower, E.H. (1971) Thyroid Status and Lipoprotein Pattern. In: Escamila, R.F., Ed., Laboratory Tests in Diagnosis and Investigation of Endocrine Function, 2nd Edition, F.A. Davis Company, Philadelphia, 101-111.

[18] De Greef, W.J., Rondeel, J.M., Van-Hasteran, G.A., Klootwij, K.W. and Visser, T.J. (1992) Regulation of TRH Production and Release in Rats. Acta Medica Australia, 19, 77-79.

[19] Hage, M., Zantout, M.S. and Azar, S.T. (2011) Thyroid Disorders and Diabetes Mellitus. Journal of Thyroid Research, 2011, 1-7. http://dx.doi.org/10.4061/2011/439463

[20] Howard, B.V. (1987) Lipoprotein Metabolism in Diabetes Mellitus. Journal of Lipid Research, 28, 613-628.

[21] Goldberg, I.J., Le, N.-A., Paternity, J.R., Ginsberg, H.N., Lindgren, F.T. and Brown, W.V. (1982) Lipoprotein Metabolism during Acute Inhibition of Hepatic Triglyceride Lipase in Nomolgus Monkey. Journal of Clinical Investigation, 70, 1184-1192. http://dx.doi.org/10.1172/JCI110717

[22] Ginsberg, H.N. (1991) Lipoprotein Physiology in Non Diabetic and Diabetic States: Relationship to Atherogenesis. Diabetes Care, 14, 839-855. http://dx.doi.org/10.2337/diacare.14.9.839 